\title{
A PROCEDURE FOR DETERMINING OPTIMAL FACILITY LOCATION AND SUB-OPTIMAL POSITIONS
}

\author{
P.K. Dan \\ Department of Industrial Engineering \\ West Bengal University of Technology, Calcutta, India \\ dan1pk@hotmail.com
}

\begin{abstract}
This research presents a methodology for determining the optimal location of a new facility, having physical flow interaction of various degrees with other existing facilities in the presence of barriers impeding the shortest flow-path as well as the sub-optimal iso-cost positions. It also determines sub-optimal iso-cost positions with additional cost or penalty for not being able to site it at the computed optimal point. The proposed methodology considers all types of quadrilateral barrier or forbidden region configurations to generalize and by-pass such impenetrable obstacles, and adopts a scheme of searching through the vertices of the quadrilaterals to determine the alternative shortest flow-path. This procedure of obstacle avoidance is novel. Software has been developed to facilitate computations for the search algorithm to determine the optimal and iso-cost co-ordinates. The test results are presented.
\end{abstract}

\section{OPSOMMING}

Die navorsing behandel ' $\mathrm{n}$ procedure vir die bepaling van optimum stigtingsposisie vir ' $n$ onderneming met vloei vanaf ander bestaande fasiliteite in die teenwoordigheid van ' $n$ verskeidenheid van randvoorwaardes. Die prodedure lewer as resultaat suboptimale isokoste-stigtingsplekke met bekendmaking van die koste wat onstaan a.g.v. afwyking van die randvoorwaardlose optimum oplossingskoste, die prosedure maak gebruik van ' $n$ vindingryke soekmetode wat toegepas word op niersydige meerkundige voorstellings vir die bepaling van korste roetes wat versperring omseil. Die prosedure word onderskei deur programmatuur. Toetsresultate word voorgehou. 


\section{INTRODUCTION}

The problems of locating a new manufacturing plant, distribution warehouse or similar facilities always involve the consideration of transport costs, which are dependent on the distance involved in moving materials between such a new facility and other existing facilities with which it has logistic interactions, such as a raw materials depot, a feeder factory, or a customer's warehouse. The distances between the new facility and each of the existing facilities determines the optimal new facility location such that the total transportation cost is minimised. Alternatively, if the location so determined is not actually available owing to certain constraints, other possible locations have to be considered, despite the additional cost or penalty cost for the sub-optimal placement of the new facility. The quantum and frequency of material movement load - the weight - influences the considerations for fixing distances. Hence optimality is dependent on the weight, which is the product of the cost per unit distance of travel and frequency of trips per unit of time. The distance between facilities for material flow has been considered here on the Euclidean distance metric with a minimum summation or mini-sum objective. In many real-life situations the straight path for material flow between facilities is not available owing to the presence of forbidden regions or of barriers such as protected land, lakes, other plants, or other physical obstacles placing constraints on movement.

However, often the optimum position of a new facility may not be physically available for economic, social, or other reasons; so a sub-optimal position, even though associated with resulting penalty costs, often has to be determined. In searching for such a location, the issue of the size of the penalty, which increases because the facility cannot be placed at the computed optimal location co-ordinate, has been a major criterion. Furthermore, the specifications affecting the choice of a sub-optimal location for the new facility often determines the decision. An analytical technique has been modeled in this research to cover both the abovementioned aspects - that is, value (the cost associated with placing the facility with penalty) and the preferred zone for a sub-optimal placement of the facility, making the approach to a solution multi-criterial. The selection of the preferred zone configuration, bounded by spans in four cardinal directions, makes the facility placement process more convenient.

There are not many solution procedures for handling such location search problems involving any number and shape of barriers or forbidden areas between facilities, thus impeding direct traffic between them. For unconstrained situations - that is, when no barrier is present to obstruct the straight path between facilities - the procedure proposed by Kuhn, as referred to by Francis and White (1974), can be deployed to find the optimal location of the new facility. However, in the recent past, the facility location problems involving barriers or forbidden regions have drawn the attention of researchers in this area. Aneja et al. (1994) have dealt with the barriers and forbidden regions through an approach based on network formation in location problems, while Batta et al. (1989) proposed a solution through their approach of cell formation. Eckhardt, as mentioned by Katz et al. (1981), dealt with some problems caused by forbidden regions by using a polygonal configuration in which paths are allowed through the forbidden region but the location of the facility itself within the region is prohibited. Brady et al. (1980) deployed interactive 
graphics to solve facility location problems with a minimax objective function involving single as well as multiple new facilities in a forbidden region that had an arbitrary configuration. Katz and Cooper (1981) studied the problem of single facility location involving a Euclidean distance metric in the presence of a circular forbidden region with a minisum objective. Larson and Sadiq (1983) have solved location problems in the presence of irregular and multiple forbidden regions involving a rectilinear distance metric. Hamacher and Nickel (1994) studied the location problem involving the restrictions of a forbidden region, developing the solution algorithms for median problems in the plane. Most of the aforementioned studies consider either a single forbidden region or a restricted region of a specific shape. The objective of the present study, therefore, is to formulate a single facility location model amidst a host of existing facilities, adhering to the Euclidean distance norm and restricted by single or multiple forbidden regions. The configuration of forbidden barriers in most of the existing research is presumed to be either rectangular or circular. This model is generalized in the sense that it considers multiple forbidden barriers with arbitrary quadrilateral shapes, including rectangles or irregular polygons, covering most applications with a single solution framework. In order to bypass the barrier contour and establish a flow route between facilities, earlier approaches used a cardinal grid element search method, or cell formation, or network formation, to explore alternative flow paths. But such methods are inefficient when handling multiple barriers of different geometries that obstruct the flow path, and cannot be deployed suitably to determine the constrained shortest flow path. This research adopts a completely new approach by formulating the least-path search through the vertices of forbidden barriers, and developing an appropriate algorithmic computational methodology for a single facility location problem. The necessary generalisation has also been done by way of considering the constraints of multiple barriers with arbitrary quadrilateral contours. The solution software that has been developed, DANSORK (Dan, 2004), determines the optimal location of a new facility under both constrained and unconstrained situations, and runs on a PC.

\section{KUHN'S PROCEDURE FOR DETERMINING OPTIMAL LOCATION}

The modality of the distance between any two location points immediately presents itself as the module of distance, expressed as a straight line or 'Euclidean' distance. If the co-ordinates for the new facility are considered to be $x_{m}$ and $y_{m}$, and those for the existing facility $e_{i}$ are chosen to be $x_{e i}$ and $y_{e i}$, so that $P=\left(x_{m}, y_{m}\right)$ and $E_{i}=\left(x_{e i}\right.$, $\left.\mathrm{y}_{\mathrm{e}}\right)$, then the Euclidean distance between $\mathrm{P}$ and $\mathrm{E}_{\mathrm{i}}$ is given by :

$d_{i}=d\left(P, E_{i}\right)=\left[\left(x_{m}-x_{e i}\right)^{2}+\left(y_{m}-y_{e i}\right)^{2}\right]^{1 / 2}$

In the single facility location problem, the objective criteria will be taken to determine the location of the new facility - say $P=\left(x_{m}, y_{m}\right)$, which will minimize the total transportation cost function $f\left(x_{m}, y_{m}\right)$, based on the adoption of the Euclidean distance norm for all the facility locations. Thus the objective of the location decision problem can be formulated as: 
$f\left(x_{m}, y_{m}\right)=\sum_{e i=1}^{n} w_{i}\left[\left(x_{m}-x_{e i}\right)^{2}+\left(y_{m}-y_{e i}\right)^{2}\right]^{1 / 2}$

where $w_{i}$ denotes the interacting weight between an existing facility and the new facility.

The two-tuple approach based on Kuhn's modified gradient procedure provides the necessary solution. The two-tuple, $T\left(x_{m}, y_{m}\right)$ is defined for all points in the plane. The established, necessary and sufficient condition for $\left(x_{m}, y_{m}\right)$ to be a minimum cost new facility location is

$T\left(x_{m}, y_{m}\right)=(0,0)$

The two-tuple can be manipulated to provide the basis for a computational procedure for determining the location $\left(x_{m}, y_{m}\right)$, and consequently the following expressions will be obtained:

$x_{m} \sum_{e i=1}^{n} w_{i} / D_{m e}=\sum_{e i=1}^{n} w_{i} x_{e i} / D_{m e}$

and $y_{m} \sum_{e i=1}^{n} w_{i} / D_{m e}=\sum_{e i=1}^{n} w_{i} y_{e i} / D_{m e}$

where $D_{m e}=\left[\left(x_{m}-x_{e i}\right)^{2}+\left(y_{m}-y_{e i}\right)^{2}\right]^{1 / 2}$

letting, $g_{i}\left(x_{m}, y_{m}\right)=w_{i} / D_{m e}$

where, $i=1$.........n

The equations are transformed to:

$x_{m}=\sum_{e i=1}^{n} x_{e i} g_{i}\left(x_{m}, y_{m}\right) / \sum_{e i=1}^{n} g_{i}\left(x_{m}, y_{m}\right)$

and $y_{m}=\sum_{e i=1}^{n} y_{e i} g_{i}\left(x_{m}, y_{m}\right) / \sum_{e i=1}^{n} g_{i}\left(x_{m}, y_{m}\right)$

\section{DISTANCE COMPUTATION INVOLVING QUADRILATERAL BARRIERS}

The minimum distance between existing facilities and a new facility in the presence of a quadrilateral obstacle (as shown in Figure 1) has been either the path connecting the points $\left(x_{e}, y_{e}\right) ;\left(x_{2}, y_{2}\right) ;\left(x_{m}, y_{m}\right)$ - that is, through one vertex point of the barrier quadrilateral, where the distance is

$d_{1}=\left[\left(x_{e}-x_{2}\right)^{2}+\left(y_{e}-y_{2}\right)^{2}\right]^{1 / 2}+\left[\left(x_{2}-x_{m}\right)^{2}+\left(y_{2}-y_{m}\right)^{2}\right]^{1 / 2}$ 
or, the path connecting the points $\left(x_{e}, y_{e}\right) ;\left(x_{4}, y_{4}\right) ;\left(x_{3}, y_{3}\right) ;\left(x_{m}, y_{m}\right)$ - that is, through two adjoining vertices of the barrier quadrilateral, where the distance is

$$
d_{2}=\left[\left(x_{e}-x_{4}\right)^{2}+\left(y_{e}-y_{4}\right)^{2}\right]^{1 / 2}+\left[\left(x_{4}-x_{3}\right)^{2}+\left(y_{4}-y_{3}\right)^{2}\right]^{1 / 2}+\left[\left(x_{3}-x_{m}\right)^{2}+\left(y_{3}-y_{m}\right)^{2}\right]^{1 / 2}
$$

The minimum of $d_{1}$ or $d_{2}$ is the shortest path between an existing facility at a fixed position and the new facility at any arbitrary location. Either of the two paths will be treated as the shortest, where $d_{1}=d_{2}$.

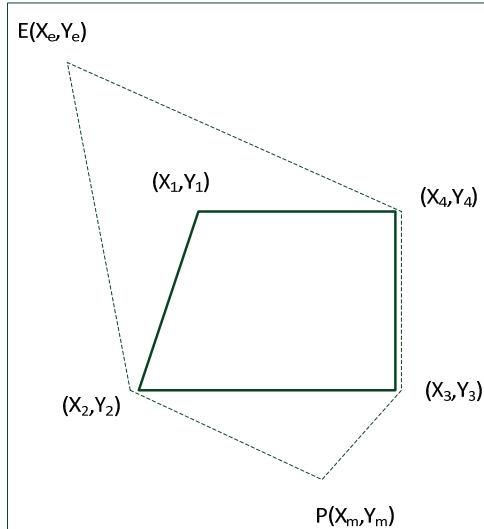

(a) Minimum path through single vertex

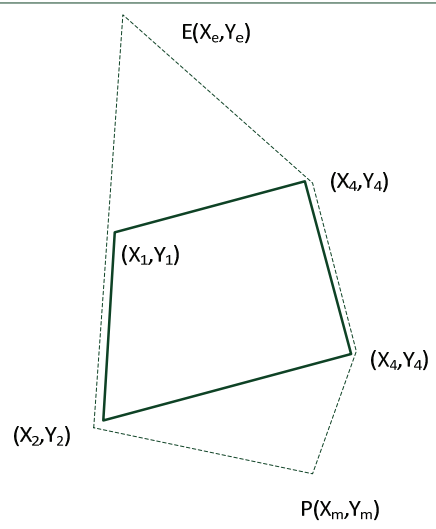

(a) Minimum path through adjoining vertices

Figure 1: Least-path distance search scheme for quadrilateral barrier

To prevent complex computations in the second case - that is, computing path length through two adjoining vertices of the barrier - a simplified distance computation procedure with a degree of approximation has been adopted, which will still produce reasonably accurate results in practice. The distance in this case is the sum of two distance segments, namely, the distance from the existing facility to the nearest vertex of the obstructing quadrilateral, and from the same vertex to the new facility located at some arbitrary point. This is the approximate substitution of the combination of three distance segments, namely, the distance of the existing facility from the nearest vertex of the obstructing quadruple, the distance of the new facility from its nearest vertex, and the distance between these two vertices. The justification is supported experimentally through three hundred problem samples. Most of the distance computations derived from the simulated experiment are oriented with the involvement of a single vertex of the barrier quadrilateral, where the need of such approximation is absent altogether; while in other, fewer cases, the distance computations involve consideration of the adjoining vertices where the aforementioned approximation will be necessary. Thereby the overall effect of such an approximation error is minimised; in fact, it is within one percent, as has been observed in the results of this experiment. 


\section{A NEW METHODOLOGY FOR DISTANCE COMPUTATION ON THE IDENTIFICATION OF BARRIERS}

\subsection{Identification of obstructive quadrilateral}

In order to establish a relationship between an existing facility and the corresponding obstructive quadrilateral impeding a straight path to the new facility, a mathematical identification of the particular barrier is necessary for softwarebased iterative computation. If a quadrilateral obstructs the straight line between a new and an existing facility, then logically the line has to intercept at least two arms of that polygon. It is imperative to check mathematically whether the intersection points are on or within the polygon arm segment. The polygon would be treated as an obstacle if more than one such intersection point is obtained for any particular polygon. The mathematical equation of a polygon arm can be expressed in the general form thus;

$a x+b y+c=0$

where $x$ and $y$ are cardinal variables, and $a, b, c$ are coefficients. Such coefficient values $[a ; b ; c]$ for each arm of a particular polygon connecting two vertices $\left(x_{s}, y_{s}\right)$ and $\left(\mathrm{x}_{\mathrm{t}}, \mathrm{y}_{\mathrm{t}}\right)$ would be given by: $\left[\left(\mathrm{y}_{\mathrm{t}}-\mathrm{y}_{\mathrm{s}}\right) /\left(\mathrm{x}_{\mathrm{t}}-\mathrm{x}_{\mathrm{s}}\right) ;-1 ; \mathrm{y}_{\mathrm{s}}-\left(\mathrm{y}_{\mathrm{t}}-\mathrm{y}_{\mathrm{s}}\right) /\left(\mathrm{x}_{\mathrm{t}}-\mathrm{x}_{\mathrm{s}}\right){ }^{*} \mathrm{x}_{\mathrm{s}}\right]$

Similarly, the coefficients [a'; b'; c'] for the line equation joining the new facility location point

$\left(x_{m}, y_{m}\right)$ and each of the existing facility location points $\left(x_{e}, y_{e}\right)$ would be given by $\left[\left(y_{m}-y_{e}\right) /\left(x_{m}-x_{e}\right) ;-1 ; y_{e}-\left(y_{m}-y_{e}\right) /\left(x_{m}-x_{e}\right)^{*} x_{e}\right]$.

The coefficients of line equations for each arm of all polygons, as well as of lines joining the new facility and each of the existing facilities, are computed using developed software. The aforementioned intersection points, $x_{\text {int }}$ and $y_{\text {int }}$, are derived as follows:

$\left[x_{\text {int }} ; y_{\text {int }}\right]=\left[\left(c^{\prime}-c\right) /\left(a-a^{\prime}\right) ;\left(c^{\prime} a-a^{\prime} c\right) /\left(a-a^{\prime}\right)\right]$

subject to the following sets of conditions:

$\left(\begin{array}{lll}x_{s} & x_{\text {int }} & x_{t}\end{array}\right)$ or $\left(x_{s}<x_{\text {int }}<x_{t}\right)$; while $\left[\begin{array}{lll}\left.y_{s} \text { or t }\right) & y_{\text {int }} & y_{t(\text { or } s)}\end{array}\right]$ or $\left[y_{s(\text { or } t)}<y_{\text {int }}<y_{t(\text { or s })}\right]$ and

$\left(\begin{array}{lll}x_{e} & x_{i n t} & x_{m}\end{array}\right)$ or $\left(x_{e}<x_{i n t}<x_{m}\right)$; while $\left[\begin{array}{lll}\left.y_{e} \text { or } m\right) & y_{\text {int }} & y_{m(\text { or e })}\end{array}\right]$ or $\left[y_{e}(\right.$ or $m)<y_{\text {int }}<y_{m}$ (or e) $]$

A polygon is treated as an obstacle for the particular existing facility if the above conditions are satisfied together. This procedure is repeated successively for all obstacles for every existing facility. The next step is to compute the minimum distance involved in bypassing the polygon, in case it has been identified as an obstacle. This step follows. 


\subsection{A methodology for computation of minimum distance in the presence of obstacle}

Lines joining a new facility and all four vertex points of the particular obstructive polygon generate equations of two lines that are tangential to the polygon at two vertices, and two other lines that will intersect the polygon. So the next computational step is to identify a pair of tangent vertices out of all four in a polygon. This is accomplished by following a similar procedure to that adopted for the identification of obstacles. Here, the coefficients [ $a$ " ; b" ; c"] of a line equation joining a vertex $\left(x_{s}, y_{s}\right)$ of the obstructive polygon and the new facility point $\left(x_{m}, y_{m}\right)$ is given by; $\left[\left(y_{m}-y_{s}\right) /\left(x_{m}-x_{s}\right) ;-1 ; y_{s}-\left(y_{m}-y_{s}\right) /\left(x_{m}-x_{s}\right)^{*} x_{s}\right]$, and the intersection point produced by this line with one of the polygon arms would be given by:

$\left[\mathrm{x}_{\text {int' }} ; \mathrm{y}_{\text {int' }}\right]=\left[\left(\mathrm{c}^{\prime \prime}-\mathrm{c}\right) /(\mathrm{a}-\mathrm{a}) ;(\mathrm{c}\right.$ "a-a"c) $/(\mathrm{a}-\mathrm{a}$ ") $)$

subject to

$\left(\begin{array}{lll}x_{s} & x_{\text {int }} & x_{t}\end{array}\right)$ or $\left(x_{s}<x_{\text {int' }}<x_{t}\right)$, while, $\left[\begin{array}{lll}\left.y_{s} \text { or t }\right) & y_{\text {int' }} & y_{t(\text { or } s)}\end{array}\right]$ or $\left[y_{s(\text { or } t)}<y_{\text {int }}{ }^{\prime}<y_{t(\text { or } t)}\right]$

where $s$ and $t$ are the terminal points of the line. Any line joining one of the vertices and the new facility would intersect one of the arms of the polygon in the case that the particular vertex is not a tangent vertex. Mathematically, in such a case the number of intersections computed would be three, including two where it is on the same point at the vertex that is the common point lying on two intersecting arms of the polygon. This common point, mathematically, is counted twice, and therefore in the case of a tangent the number of such intersections is two. A counter for the computed number of such intersections has been provided in the software. By connecting the two tangent points of the polygon to the location point of the existing facility, two separate paths with computed distances following the approximation technique (referred to in section (3)) are obtained and the minimum of these is selected. The mathematical expression of minimum distance through the corresponding polygon vertex points $\left(x_{s k}, y_{s k}\right)$ of the $j^{\text {th }}$ obstacle at the $k^{\text {th }}$ vertex from the new facility point $\left(x_{m}, y_{m}\right)$ to the existing $i^{\text {th }}$ facility point $\left(x_{e i}, y_{e i}\right)$ is:

Distance $=\left[\left(\mathrm{x}_{\mathrm{jk}}-\mathrm{x}_{\mathrm{m}}\right)^{2}+\left(\mathrm{y}_{\mathrm{jk}}-\mathrm{y}_{\mathrm{m}}\right)^{2}\right]^{1 / 2}+\left[\left(\mathrm{x}_{\mathrm{jk}}-\mathrm{x}_{\mathrm{ei}}\right)^{2}+\left(\mathrm{y}_{\mathrm{jk}}-\mathrm{y}_{\mathrm{ei}}\right)^{2}\right]^{1 / 2}$

The summation of the products of the distances and relative weights $\left(w_{i}\right)$ provides the total cost $(C)$ Such arithmetic products are computed for both the constrained (that is, in the presence of barriers) and the unconstrained (free of barrier) situations. The total cost $(C)$ burden for the particular location of the new facility can be expressed as:

$$
\begin{gathered}
C=\sum_{\text {constrained }} w_{i}\left\{\left[\left(x_{j k}-x_{m}\right)^{2}+\left(y_{j k}-y_{m}\right)^{2}\right]^{1 / 2}+\left[\left(x_{j k}-x_{e i}\right)^{2}+\left(y_{j k}-y_{e i}\right)^{2}\right]^{1 / 2}\right\}+ \\
\sum_{\text {unconstrained }} w_{i}\left[\left(x_{m}-x_{e i}\right)^{2}+\left(y_{m}-y_{e i}\right)^{2}\right]^{1 / 2}
\end{gathered}
$$




\subsection{Cardinally explorative search procedure for optimal location determination}

The new facility, at any sub-optimal or optimal situation - say, at an arbitrary location $\mathrm{P}$ - can physically be any point within the spatial boundary of existing facilities $E_{i}$, located at different coordinate points in the cardinal plane, and $d^{*}$ is the effective distance. For optimality searching, any coordinate point representing the new facility may be selected as the starting point for the search. Assuming that the starting point is $\left(\mathrm{x}_{0}, \mathrm{y}_{0}\right)$ for the new facility in the chosen plane, having a corresponding value of cost burden $(C)$ as $C_{0}$

where $C=\sum_{e i=1}^{n} w_{i} d^{*}\left(P, E_{l}\right)$

the next step is to check the value of $C$, 1-unit apart in all four cardinal directions ( 2 on the abscissa and 2 on the ordinate) - namely, at $\left(x_{0}, y_{0}+1\right) ;\left(x_{0}+1, y_{0}\right) ;\left(x_{0}, y_{0}\right.$ $1)$; $\left(x_{0}-1, y_{0}\right)$. Suppose that in the first iteration the minimum value at any of the above cardinal points is $C_{1}$. Then in the next iteration, the coordinate corresponding to the above minimum $C$ (i.e, $C_{1}$ ) will be treated as the fresh starting point for the next iteration, and so on until the value of $C$ converges to a minimum and the coordinate point $\left(x_{m}, y_{m}\right)$, corresponding to such minimum value, is the optimal location point of the new facility. This algorithmic technique, developed in the optimality modeling software with graphical representation, is oriented with a cardinal exploration-based search through a converging series of locational values.

\subsection{Procedure for determination of iso-cost positions}

The search zone has been defined in the present research with four cardinal spans around a reference point, which usually is the computed optimal position of a new facility. Such span or spread is expressed in 'Cartesian' mapping-units in northerly, southerly, easterly, and westerly directions to describe a rectangular zone having a number of grid points with unit-distance spacing. Therefore, the equal cost (or isocost) search zone is defined through a pair of cardinal spans along the x-axis and the $y$-axis. The iso-cost values are settled with a range instead of a single value, since the value searching process uses discrete integer grid co-ordinate points only, and therefore a specific value with a decimal component may not actually occur. The value at each grid point is derived from a least-distance computation technique (as has already been modeled here), and values satisfying the upper and lower bound conditions are recorded for iso-cost band searching. Since the values involve a range, a curve in the form of a band would emerge instead of a linear curve. The values of the test results with respect to the alternative equal cost or iso-cost locations are arranged in an ascending order of value in the computerized output, following the selected range of desired or acceptable costs associated with the placement of a new facility within the specified configuration of the iso-cost search zone. Computational parameters for iso-cost determination and the corresponding results are presented in the following section. 


\section{SALIENT FEATURES OF THE DEVELOPED ANALYTICAL SOFTWARE}

The software for the analytical solution of the location problem includes a graphical representation of the optimality framework for use on a PC, and is structured on integrated functional modules.

Data entry: For various inputs which are necessary for defining the problem conditions associated with a new facility location analysis, pertaining to the coordinate location of facilities, obstacle locations, inter-facility load-flow weights, and search iteration starting point. Based on the data entry sequence, the existing facilities are numbered E1, E2, E3, etc. The software does this numbering automatically. The quadrilateral obstacle vertices are numbered F011, F012, F013, and F014, where the $\mathrm{F}$ denotes the forbidden barrier, the first two digits represent the obstacle reference number, and the third digit indicates each vertex number of that specific quadrilateral. This needs to be entered in a sequential order for defining the barrier configuration. The iteration starting reference coordinate point is also entered as the operating input data.

\section{RESULTS OF THE EXPERIMENT}

A sample problem was set for experimental study involving six existing facilities under an unconstrained condition - that is, without any impenetrable barrier. Subsequently a problem with five quadrilateral barriers was considered. They are described in the next three sub-sections.

6.1 The solution based on Kuhn's procedure for the following problem is presented here.

\begin{tabular}{ccc}
$\begin{array}{c}\text { Existing facility serial } \\
\text { number }\end{array}$ & $\begin{array}{c}\text { Coordinate locations of } \\
\text { existing facilities }\end{array}$ & $\begin{array}{c}\text { interacting weight with } \\
\text { respect to new facility }\end{array}$ \\
\hline 1 & $(47,4)$ & 0.15 \\
2 & $(50,33)$ & 0.15 \\
3 & $(45,47)$ & 0.20 \\
4 & $(6,45)$ & 0.20 \\
5 & $(0,1)$ & 0.20 \\
6 & $(30,2)$ & 0.10
\end{tabular}

The starting point $\left(\mathrm{x}_{0}, \mathrm{y}_{0}\right)$ has been determined using the squared Euclidean distance metric (as is common practice), and its value is $(27.75,24.35)$. The results below show convergence after each iteration:

$\begin{array}{ccc}\text { Coordinates } & \text { Mini-sum values } & \text { Status } \\ \left(\mathrm{x}_{0}, \mathrm{y}_{0}\right):(27.75,24.35) & 28.9740 & \text { Starting } \\ \left(\mathrm{x}_{1}, \mathrm{y}_{1}\right):(29.82,24.70) & 28.8500 & \\ \left(\mathrm{x}_{2}, \mathrm{y}_{2}\right):(30.88,25.00) & 28.8160 & \\ \left(\mathrm{x}_{3}, \mathrm{y}_{3}\right):(31.44,25.33) & 28.8050 & \\ \left(\mathrm{x}_{4}, \mathrm{y}_{4}\right):(31.78,25.47) & 28.8020 & \\ \left(\mathrm{x}_{5}, \mathrm{y}_{5}\right):(31.87,25.61) & 28.8010 & \text { Optimum } \\ \left(\mathrm{x}_{6}, \mathrm{y}_{6}\right):(31.87,25.61) & 28.8006 & \end{array}$


The experimental study of the optimality search results using DANSORK software for the same problem is presented in this section. The search iteration starting point is in the middle, that is, at the coordinate location $(25,25)$, as the problem is formatted in a 50-unit square grid space. The iterative search of this unconstrained problem, and the corresponding coordinate location of the new facility, are as follows:

\begin{tabular}{|ccc|}
\hline Coordinates & Mini-sum values & Status \\
\hline 25,26 & 29.25 & Starting \\
\hline 26,25 & 29.13 & \\
\hline 27,25 & 29.03 & \\
\hline 28,25 & 28.95 & \\
\hline 29,25 & 28.89 & \\
\hline 30,25 & 28.84 & \\
\hline 31,25 & 28.82 & Optimum \\
\hline 32,25 & 28.81 & \\
\hline 32,26 & 28.80 & \\
\hline
\end{tabular}

An experimental sample problem-set, involving the six existing facilities constrained by five forbidden barriers, is presented in this section. The results of an optimality search using the software is also presented, along with the graphical representation of the connecting paths. The problem is formatted in a $50 * 50$ grid space.

Experimental problem-set: Parameters under constrained facility location situations

Barrier Number

20

Co-ordinate of vertices of quadrilateral barrier

$\begin{array}{ll}1 & (10,39) ;(20,40) ;(20,42) ;(10,43) \\ 2 & (10,5) ;(15,5) ;(15,11) ;(12,11) \\ 3 & (42,5) ;(46,5) ;(45,11) ;(43,11) \\ 4 & (28,7) ;(31,4) ;(34,7) ;(31,10) \\ 5 & (40,42) ;(42,37) ;(46,42) ;(43,44)\end{array}$

Computerized output of analytical software (with reference coordinate point for initiating optimality search iteration at 25, 25 chosen arbitrarily)

\begin{tabular}{|ccc|}
\hline Initial reference point & 2525 & 29.43 \\
\hline Point 1 & 2526 & 29.46 \\
\hline Point 2 & 2625 & 29.34 \\
\hline Point 3 & 2524 & 29.42 \\
\hline Point 4 & 2425 & 29.54 \\
\hline Next reference point & 2625 & 29.34 \\
\hline
\end{tabular}


A graphical representation of the constrained inter-facility connecting path at optimality condition for the problem set is depicted in Figure 2.

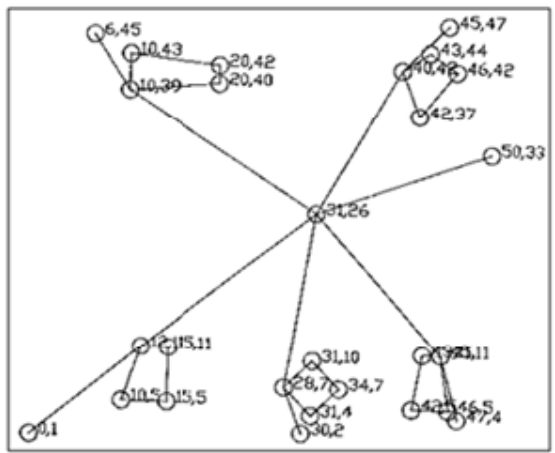

Figure 2: Graphical representation of the construction inter facility connecting path at optimality condition.

Summary of results:

\begin{tabular}{|ccc|}
\hline & Coordinate & Value \\
\hline $\mathbf{1}$ & 2625 & 29.43 \\
\hline $\mathbf{3}$ & 2725 & 29.27 \\
$\mathbf{4}$ & 2825 & 29.20 \\
$\mathbf{5}$ & 2925 & 29.15 \\
$\mathbf{6}$ & 3025 & 29.12 \\
\hline $\mathbf{7}$ (Optimal) & 3026 & 29.11 \\
\hline $\mathbf{8}$ & 3126 & 29.10 \\
\hline
\end{tabular}

Parametric conditions for iso-cost location analysis:

Search with reference location at $(31,26)$

Defined iso-cost zone with select values:

\begin{tabular}{cc|}
$\begin{array}{c}\text { Cardinal direction with respect } \\
\text { to reference point (in grid units) }\end{array}$ & $\begin{array}{c}\text { Cardinal } \\
\text { coverage span }\end{array}$ \\
\hline North & 10 units \\
East & 10 units \\
South & 20 units \\
West & 10 units \\
\hline
\end{tabular}

Formulated experimental range for iso-cost determination as +0.05 or -0.05 from a value of 32 (i.e, values between 31.95 and 32.05). 


\section{Computer output of the iso-cost location results}

$\begin{array}{ccc}X & Y & \text { Value } \\ 14 & 19 & 31.95 \\ 14 & 30 & 31.95 \\ 15 & 16 & 31.96 \\ 15 & 33 & 31.99 \\ 14 & 18 & 32.03 \\ 14 & 31 & 32.04 \\ 13 & 24 & 32.05 \\ 13 & 25 & 32.05\end{array}$

\section{CONCLUSION}

The results for the optimality condition for the corresponding iso-cost value, along with the location coordinates for the unconstrained problem - determined by using both Kuhn's procedure and the developed algorithmic solution procedure - show no real difference. The validity testing of the computational software is established by comparing the results in Section 6.1 and Section 6.2. Subsequently the functionality of the software has been extended to cover the constrained condition - that is, involving barriers. Manual computation provides exactly the same value as that obtained with the DANSORK software for the constrained problem condition illustrated in Section 6.3. This establishes that the software can be deployed, along with the new obstacle search algorithm for both unconstrained and constrained conditions of facility location.

\section{REFERENCES}

[1] Aneja, Y.P., \& Parlar, M. 1994. Algorithm for Weber facility location in the presence of forbidden regions and/or barriers to travel. Transportation Sciences, Vol. 28, No.1.

[2] Batta, R., Ghosh, A., \& Palekar, U.S. 1989. Locating facilities on the Manhattan metric with arbitrary shape barriers and convex forbidden regions. Transportation Sciences, Vol. 23, No.1.

[3] Brady, S.D., \& Rosenthal, R.E. 1980. Interactive graphical solutions of constrained minimax location problems. AllE Transactions, 12, 241-248.

[4] Dan, P.K. 2004. A new computerized solution procedure for generalized facility location problem. $33^{\text {rd }}$ International Conference on Computers and Industrial Engineering, South Korea.

[5] Francis, R. L. \& White, J.A. 1974. Facility layout and location: An analytical approach. N.J.: Prentice Hall.

[6] Hamacher, H.W. \& Nickel, S. 1994. Combinatorial algorithms for some 1facility median problems in the plane. European Journal of Operational Research, 79, 340-351. 
[7] Katz, I.N. \& Cooper, L. 1981. Facility location in the presence of forbidden regions: Formulation and the case of a Euclidean distance with forbidden circle. European Journal of Operations Research, 6, 166-173.

[8] Larson, R.C. \& Sadiq, G. 1983. Facility locations with the Manhattan metric in the presence of barriers to travel. Operations Research, 31, 652-669. 
http://sajie.journals.ac.za 